\title{
On storm weakening during substorm expansion phase
}

\author{
G. L. Siscoe, H. E. Petschek \\ Center for Space Physics, Boston University, Boston, Massachusetts, USA
}

Received: 8 March 1996 / Revised: 31 July 1996 / Accepted: 6 August 1996

\begin{abstract}
Iyemori and Rao recently presented evidence that the strength of a magnetic storm, as measured by -Dst, weakens, or its rate of growth slows, during the substorm expansion phase. Yet the expansion phase is known to inject energetic particles into the ring current, which should strengthen the storm. We propose to reconcile these apparently contradictory results by combining the virial theorem and a principle of energy partitioning between energy storage elements in a system with dissipation. As applied to the unloading description of the substorm expansion phase, the virial theorem states that -Dst is proportional to the sum of the total magnetic energy and twice the total kinetic energy in the magnetosphere including the tail. Thus if expansion phase involves converting magnetic energy stored in the tail into kinetic energy stored in the ring current, a drop in -Dst during expansion phase requires that less than half the drop in magnetic energy goes into the ring current, the rest going into the ionosphere. Indeed Weiss et al., have estimated that the energy dissipated in the ionosphere during expansion phase is twice that injected into the ring current. This conclusion is also consistent with the mentioned energy partitioning principle, which requires that more energy be dissipated than transferred between storage elements. While Iyemori and Rao's observations seem to contradict the hypothesis that storms consist at least in part of a sum of substorms, this mode of description might nonetheless be preserved by including the substorm's growthphase contribution. Then the change in storm strength measured from before the growth phase to after the expansion phase is positive, even though the expansion phase alone makes a negative contribution.
\end{abstract}

\section{Must a substorm be a sub-storm?}

For about two decades some of our colleagues have been saying that the sub-storm hypothesis, which is that a magnetic storm is composed of a series of substorms, is too simplistic if not false (for an early instance, see Kamide, 1980; for a strong version of this position, see Kamide, 1992; for a review, see Gonzalez et al., 1994). An observation bearing on this issue was recently reported. Results published by Iyemori and Rao (1996) show that, as measured by -Dst, a storm's strength or its rate of strengthening decreases during a substorm expansion phase. Specifically, they find that on average, during a magnetic storm's main phase, -Dst rises more slowly after an expansion phase starts than before, and that on average, during a storm's recovery phase, it drops more rapidly. To guard against being misled by timing uncertainties, they use multiple criteria to time the start of the substorm expansion phase: midlatitude positive bays, sharp decreases in the AL index, and Pi2 onsets. In each case the storm or its growth weakens.

The Iyemori and Rao result appears to test the hypothesis that gave substorms their name. As Chapman conceived it in a 1962 article, "A magnetic storm consists of sporadic and intermittent disturbances.... These I call polar substorms." (quote taken from Kamide, 1992). Chapman's choice of verb, 'consists,' that is, 'is made up of,' implies that he meant the prefix sub- in the sense of a division into smaller pieces of the same kind (e.g., in the sense of subdivision and subsection). In this view, a magnetic storm is the accumulation of a number of small storms, which can therefore be called sub-storms. Since for the following discussion we need a way to distinguish between the phenomenon which now bears the name substorm and the hypothesis behind the name, we use 'sub(hyphen) storm' when we mean the hypothesis. Thus we should say that Chapman's idea was that storms are made up of a series of sub-storms.

There is a further semantic problem that needs to be addressed early on. What Chapman and after him Akasofu meant by 'substorm' was the part of the magnetospheric substorm that we now call the substorm expansion phase or the expansion and recovery phases together. This newer nomenclature is used to distinguish these active phases from the growth phase, which was identified as a separate phase after 'substorm' had 
already become part of the standard magnetospheric lexicon (McPherron, 1970). As a result, there exists still today a commonly occurring ambiguity in which people use substorm in both senses: as meaning a three-phased phenomenon and a two-phased phenomenon. Usually the ambiguity does no harm, that is, it does not impede communication. For the purpose of the subject of this paper, however, the distinction might be crucial, as will be seen. Thus, to remove the ambiguity, we will use 'substorm (2)' to mean the original hypothesis, which referred to a two-phased phenomenon. Then we can say without ambiguity that the Iyemori and Rao result is counter to the sub-storm (2) hypothesis. (Recall that they find that during the substorm expansion phase, magnetic storms as measured by -Dst weaken or their growth slows.) But, as we will see, the Iyemori and Rao result might not be counter to a 'sub-storm (3)' hypothesis, where (3) means the three-phased version of the phenomenon.

The Iyemori and Rao result, which contradicts the sub-storm (2) hypothesis, leaves us with a problem. We know that during its expansion phase a substorm injects energetic particles into the magnetosphere which should contribute to strengthening the storm. This being so, how can substorms not satisfy the sub-storms (2) hypothesis? A possible response to the apparent clash of observations is that the Iyemori and Rao result, or its interpretation, is somehow wrong. This possibility must be resolved by others repeating the test or offering alternative interpretations. The correctness of the observation or its interpretation is not the issue we address here. Instead we take the Iyemori and Rao result as having raised an interesting question that has not been posed before, and which we will address: Is there a substorm scenario that entails no sub-storm (2), that is, no strengthening of the storm during the expansion phase? We find the answer to be, yes. A non-sub-storm (2) substorm scenario turns out to be the familiar unloading scenario for the expansion phase (e.g., Baker, 1992) with the added condition that of the energy unloaded, more goes into the ionosphere than into injected particles.

The main point of this paper is to demonstrate that the unloading substorm expansion phase scenario can be a non-sub-storm (2) scenario. For the demonstration we combine two general principles: the virial theorem and a principle of partitioning energy between the storage and dissipation elements of an electrodynamical system. The virial theorem as applied to the unloading substorm expansion phase model states that under general conditions the change in -Dst is proportional to the sum of the change in the total magnetic energy and twice the change in the total thermal energy. In both cases, 'change in total energy' refers to all the energy inside a volume that includes the magnetosphere and the tail. Thus in the unloading substorm expansion phase model, when stored magnetic energy in the tail converts to thermal energy in the magnetosphere, the drop in magnetic energy (so called dipolarization) weakens -Dst (weakens means to reduce it or to slow its growth), while the gain in thermal energy (particle injection) strengthens it. Because of the factor of 2 multiplying the thermal-energy term in the -Dst equation, if all the magnetic energy lost in dipolarization goes into energizing particles and injecting them into the magnetosphere, the net effect of dipolarization and injection should be to strengthen -Dst. Under this condition, the sub-storm (2) hypothesis should work.

The stated condition of full conversion of magnetic energy into injected particle energy need not happen, however, for some of the stored magnetic energy lost in dipolarization goes into the ionosphere as Joule heat and particle precipitation. The energy lost to the ionosphere does not contribute to strengthening -Dst. Thus, whether a substorm expansion strengthens or weakens -Dst depends on how the energy released by dipolarization is partitioned between particles injected into the magnetosphere and heat dissipated in the ionosphere. If more energy goes into the magnetosphere, there should be net strengthening of -Dst. If more goes into the ionosphere, there should be net weakening.

A published estimate, based on observations, suggests that during substorms more energy goes into the ionosphere than into the magnetosphere (Weiss et al., 1992). Also as shown below, functionally analogous lumped circuits exhibit the general property that more energy goes into Joule heat (the ionosphere) than into storage (the ring current). If valid, these indications imply that the substorm expansion phase should cause a net weakening of -Dst, as Iyemori and Rao observe.

The following sections document statements made in this opening summary.

\section{The virial theorem applied to the sub-storm hypothesis}

Baker and Hurley (1967) used a virial-theorem approach to find an upper limit on strength of the Van Allen belts. Olbert et al. (1968), Siscoe (1970), and Carovillano and Siscoe (1973) extended the approach to obtain a generalized form of the Dessler-Parker-Sckopke equation, which relates Dst to various global magnetospheric energies (Dessler and Parker, 1959; Sckopke, 1966). To address the Iyemori and Rao question, we use the generalized form of the DPS equation.

The power of the DPS equation is its generality. Dessler and Parker derived a specialized version of the equation based on particles with either zero- or ninetydegree pitch angles and a dipole magnetic field. Sckopke showed that their specialized version holds also for arbitrary pitch angles, though still with a dipole magnetic field. Baker and Hurley generalized the equation to include the self field of the particles. The subsequent generalizations included also the fields generated by boundary currents including the tail.

The generalized DPS equation can be written as [cf., Siscoe (1970) Eq. 24 in which the terms from internal and boundary sources are combined]

$\frac{B_{m}(0)}{B_{0}}=-\frac{2 K+M-\oint \boldsymbol{R} \cdot \hat{\boldsymbol{n}} d \sigma}{3 M_{D}}$. 
Before defining the symbols, we note that the surface over which the integral is to be taken is arbitrary. We take it to be the magnetopause (its inner surface) which is closed by a crossection placed far enough down the tail that the magnetic field at the earth generated by all currents beyond it is negligible. This choice lets us call the volume so enclosed the magnetosphere. Then the symbols have the following meanings: $B_{m}(0)$ is the component at the earth's dipole and antiparallel to it of the combined magnetospheric magnetic field from all sources (ring current, magnetopause, and tail); $B_{0}$ is the equatorial strength of the dipole field at the earth's surface; $K$ is the total thermal energy of all charged particles in the magnetosphere (i.e., in the ring current and the plasma sheet inside the defining volume); $M$ is the total magnetospheric magnetic energy inside the magnetosphere (i.e., the magnetospheric volume integral of $B_{m}{ }^{2} / 2 \mu_{\mathrm{o}}$, where $B_{m}$ is the sum of the fields generated by the boundary currents, the tail current, and the ring current); $M_{D}$ is the energy in the earth's dipole field above the earth's surface, and $\boldsymbol{R}$ is given by [cf., Siscoe (1970) Eq. 8]

$\boldsymbol{R}=\left(p+\frac{B^{2}}{2 \mu_{0}}\right) \boldsymbol{r}+\rho(\boldsymbol{V} \cdot \boldsymbol{r}) \boldsymbol{V}-\frac{(\boldsymbol{B} \cdot \boldsymbol{r}) \boldsymbol{B}}{\mu_{0}}$.

Here $p$ is the thermal pressure (assumed isotropic); $\boldsymbol{r}$ is the radius vector from the center of the earth; $\rho$ is the mass density of the solar wind in the mantel (assuming this to lie within the magnetopause); $\boldsymbol{V}$ is the velocity of the solar wind in the mantle; and $\boldsymbol{B}$ is the total magnetic field (dipole plus magnetospheric). There is a corresponding expression for anisotropic pressure, but it is not needed here. Finally, the unit vector in Eq. 1 is the outward pointing normal.

As in all derivations of the DPS equation, Dst is taken to be proportional to $B_{m}(0)$. For example, in an ideal situation in which Dst is the true surface average of the component of the perturbation field antiparallel to the dipole axis, and in which there is perfect shielding of the perturbation field by ground-induced currents (the case often assumed), Dst $=3 / 2 B_{m}(0)$. Thus, Dst is proportional to the right-hand side of Eq. 1.

For the following reasons, we may drop the surface integral when we apply Eq. 1 to the unloading form of the sub-storm (2) hypothesis. This form says that a sub-storm is a process that releases magnetic energy stored in the tail and converts it into injected particle energy. The conversion from stored magnetic energy to stored kinetic energy (ring-current thermal energy) is the process that strengthens or builds up the storm. Since storm strength is measured by -Dst, we must recast all conclusions into statements about -Dst. The sub-storm (2) hypothesis says that the energy conversion process increases the -Dst measure of storm strength.

Now we can see how the simplification to Eq. 1 dropping the surface integral - comes about. Note that the hypothesis mentions only the conversion of $M$-type energy into $K$-type energy (e.g., Hesse, 1995). With regard to the energy budget that relates sub-storms to storms, there is nothing in any version of the unloading sub-storm (2) hypothesis that postulates a necessary involvement of processes occurring at the boundary during the expansion phase. No version taps or changes the energy flowing through the boundary as a necessary part of the sub-storm-to-storm energy budget. The hypothesis merely says that some energy comes out of $M$ and goes into $K$, and as a result Dst drops. Thus, the equation that epitomizes the unloading form of the substorm (2) hypothesis may be written as

$\frac{\Delta B_{m}(0)}{B_{0}}=-\frac{2 \Delta K+\Delta M}{3 M_{D}}$,

where the deltas denote the change in quantities before and after the unloading. This is a general result.

It might be objected that although no builduprelease scenario explicitly invokes changes at the boundary, nonetheless, such changes are entailed by the decompression associated with the release. To allay fears that the boundary term might dominate the other terms on the right-hand side of Eq. 3, we note that the main effect at the surface during decompression is a reduction in field strength and tail radius. Therefore, the ratio of the change in the surface term to the change in the magnetic energy term is roughly the ratio of the change in crossectional area to the crossectional area itself, or about 10 to 20 percent. Another point of reference is this: the surface term basically gives the tail's contribution to the positive Chapman-Ferraro field at Earth. By contrast, the focus of our discussion is the Dst field of magnetic storms. The incommensurability of the strengths of these two fields (the tail's contribution to the Chapman-Ferraro field is much smaller than the traditional contribution which is in turn much smaller than the storm-time Dst) should allow us to proceed safely with the simplification that Eq. 3 gives.

Equation 3 shows that as measured by -Dst, the quantitative change in the strength of the storm that occurs as a result of an unloading-type substorm expansion phase is proportional to the algebraic sum of the changes in $2 K$ and $M$. The strength increases if the sum is positive and decreases if it is negative. In the unloading substorm scenario, $\Delta M$ is negative and $\Delta K$ is positive. Furthermore, $\Delta K$ is some fraction of $|\Delta M|$ which depends on how much of $\Delta M$ goes into energizing and injecting particles into the magnetosphere (i.e., $\Delta K$ ), and how much goes into the ionosphere as Joule heat and particle precipitation. The equation shows that if the fraction that goes into particle injection is greater than $1 / 2$, the storm strengthens, and that if it is less than $1 / 2$, the storm weakens. This result verifies a major claim made in the introductory section.

To complete the demonstration that the unloading substorm expansion phase scenario can be a non-substorm (2) scenario, it remains to estimate the magnetosphere-to-ionosphere partitioning of the energy that is released during substorm unloading. 


\section{The branching ratio of injected to dissipated energy}

Weiss et al. (1992) have compiled a table of energies associated with storms and substorms. Their table incorporates many earlier estimates and thus gives the best synthesis of such estimates currently available. Of interest here are their entries for 'Ring Current' and Joule Heating.' They give two cases, 'Typical' and 'Large'; in both, the entry for Joule heating is twice the entry for ring current. In the text they state as a generality, "Statistical and case studies indicate that [during the expansion phase of a substorm] the predominant energy dissipation mechanism is Joule heating..." They mean explicitly that Joule heating predominates over "particle heating and plasma injection." One source they draw on for this assessment is the report of Harel et al. (1981) on a quantitative substorm simulation using the Rice Convection Model. These authors find that "global Joule heating...is about three times the change in ring current energy for the modeled substorm." They support this numerical result with an analytic estimate which generalizes the finding to virtually all substorms qualitatively (i.e., 'replace about three times' with 'greater than').

To connect the information in the Weiss et al. table which relates to $\Delta K$ (the change in ring-current energy) and $\Delta J$ (the energy lost in Joule heat) to $|\Delta M|$ (the energy given up by the magnetic field in the unloading process), we assume that $\Delta K$ and $\Delta J$ are the main sinks of $|\Delta M|$. That is, we assume that $|\Delta M|=\Delta K+\Delta J$. This assumption, which in words is that sinks of energy derived from $|\Delta M|$ other than $\Delta K$ and $\Delta J$ can be neglected, is justified by reference again to the Weiss et al. table, which, for example, sets the energy of electron precipitation at about an order of magnitude less than the energy lost to Joule heating.

With this connection between $|\Delta M|, \Delta K$, and $\Delta J$, we may conclude on the basis of the Weiss et al. synthesis and the detailed analysis of Harel et al. that $\Delta K /|\Delta M|$ $<1 / 2$, and that perhaps it is considerably smaller. For example, if we take the factor of two by which Weiss et al. estimate joule heating to exceed injection energy, we have $\Delta K /|\Delta M|=1 / 3$. If we take the factor of three from the Harel et al. numerical result: $\Delta K /|\Delta M|=1 / 4$. In all cases for which quantitative estimates are given, $\Delta K /|\Delta M|<1 / 2$. This result together with the earlier result based on the virial theorem (Eq. 3) implies that as a general rule, Dst should weaken during substorms or its rate of strengthening drop. This establishes the second main claim given in the introductory section.

Equation 3, the finding from the literature that $\Delta K /|\Delta M|<1 / 2$, and the conclusion just given that follows from these two items, constitute the main message of this paper. Nonetheless, we wish to add a further thought, which is that the finding that $\Delta K /|\Delta M|=1 / 2$ is possibly not an accident. By this we mean that the inequality is possibly not a property of substorms that is contingent on the particular magnetospheric and ionospheric parameters that the earth happens to possess and that could be otherwise if these parameters were different. We suggest that instead this inequality might be an essential attribute of the coupled magnetosphere-ionosphere system when it engages in the dynamical mode that is called substorm expansion. Stated more briefly, we pursue next the idea that the inequality $\Delta K /|\Delta M|=1 / 2$ cannot be otherwise. If it cannot be otherwise, the ability of substorm expansion phases to weaken storms as measured by -Dst, which is seen to exist in case studies and in statistical studies and which can be understood as just explained, is an intrinsic, not an accidental and, therefore, possibly occasionally violated, property of the system.

\section{The possible noncontingency of the inequality $\Delta K /|\Delta M|=1 / 2$}

Before taking up the project as stated, let us recast the inequality in form $\Delta K / \Delta J<1$, since $\Delta K$ and $\Delta J$ are the quantities to which most studies relate.

The analytical treatment of Harel et al. (1981) to some extent already establishes the point we wish to make. Their treatment uses for the energy lost to Joule heat the expression $J \Phi T$, where $J$ is the injected particles' contribution to the ring current that generates Dst, $\Phi$ is the transpolar potential, and $T$ is the time the injected particles need to develop a complete ring. The ratio of this estimate of $\Delta J$ to the particle energy that the injection adds to the ring current $(\Delta K)$ turns out in their formalism to depend merely on three parameters: the energy of the particles (through the drift time), their drift L-shell, and the transpolar potential. Although the numerical value of the energy ratio thus obtained is less than unity for all interesting values of the three quantities, their formulation does not obviously preclude values of the ratio that are greater than unity. That is, from their formulation we cannot derive the inequality $\Delta K / \Delta J<1$ as a necessary condition. It is merely a condition that happens to be satisfied for typical values of the operative parameters.

To explore another approach to the problem of calculating $\Delta K / \Delta J$, we examine the properties of functionally analogous lumped circuits. We do this despite the existence in some quarters of the strongly held view that lumped-circuit analogies in magnetospheric physics are inappropriate. We believe the opposite view can be as strongly defended [for example, by reference to the stunning success Goertz et al. (1993) achieved in modeling geomagnetic activity using only electrical-engineering principles and the useful insights Klimas et al. (1992) gleaned on the behavior of substorms through exploring the properties of the Faraday loop model], but it would be unwarranted here to launch into a major defense of what is in effect an appendix to our main result. Instead we limit the ambition of our project to be merely to define expectations. Before now there were no expectations based on general principles regarding the partitioning of energy between dissipated (Joule) and stored (ringcurrent) forms in the coupled magnetosphere-ionosphere system. Our intent is to supply a comparison by which to orient expectations by noting that lumped 
circuits with functionally analogous storage and dissipation elements exhibit as a necessary condition the property $\Delta K / \Delta J<1$. Thus, we might expect the magnetosphere-ionosphere system to exhibit the same property, and we might therefore further expect from the earlier sections that substorm expansion phases will weaken -Dst as a general rule. An expectation is, of course, not a proof, just a frame of reference or a point of departure. If subsequent research demonstrates a circumstance where $\Delta K / \Delta J>1$, it would be an event of note, something like finding an exception to Lenz's law.

We start by recalling a puzzle familiar from elementary circuit theory: if a charged capacitor is suddenly connected by closing a switch to an uncharged but otherwise identical capacitor, the sum of the energies stored in both capacitors after the connection is half the original stored energy (this is because the stored energy is proportional to the square of the charge; thus the energy in each capacitor after connection is $1 / 4$ the initial energy). Where did the missing energy go? It was dissipated as Joule heat, regardless of the value of the resistance in the connecting wire (the value of the resistance merely sets the time needed to dissipate the energy). This example is functionally analogous to our case in that it contains two storage elements and a resistor. The point is that the result is independent of the values of the capacitances and the resistance. It shows that in this case of identical storage elements, the amount of energy transferred to the second storage element equals $1 / 2$ the amount of energy dissipated. To express this result in terms of the analog symbols used above for the substorm: $\Delta K / \Delta J=1 / 2$. In the general case in which the first and second capacitors mentioned have capacitances $C_{1}$ and $C_{2}$, the result is $\Delta K / \Delta J=$ $C_{1} /\left(C_{1}+C_{2}\right)$, which is less than 1 for all non-zero values of the capacitances.

Is there an example of a lumped circuit that is not only functionally analogous to the system we have been treating but also structurally analogous? To see that there is, consider again the four elements that make up the system we have been treating: an element that stores magnetic energy (the tail which loses energy $|\Delta M|$ during the expansion phase); an element that stores kinetic energy (the ring current which gains energy $\Delta K$ ); an element that dissipates energy (the ionosphere which consumes energy $\Delta J$ ), and a switch that at the onset of the expansion phase opens to shunt current from the magnetic storage element through the kinetic storage element and the dissipation element. In the lumpedcircuit analog, the magnetic storage element is an inductor, which we take to have inductance $L_{M}$. The dissipation element is a resistor, which we take to have resistance $R$. The kinetic storage element could be either a capacitor (if the kinetic energy is in the form of flowing plasma - the usual situation treated in text books) or an inductor [if the kinetic energy is in thermal form demonstrated for the case of the region-2 current system (Siscoe, 1982)]. Since the kinetic energy in the ring current is in thermal form, we treat the case of an inductor and take its inductance to be $L_{K}$.

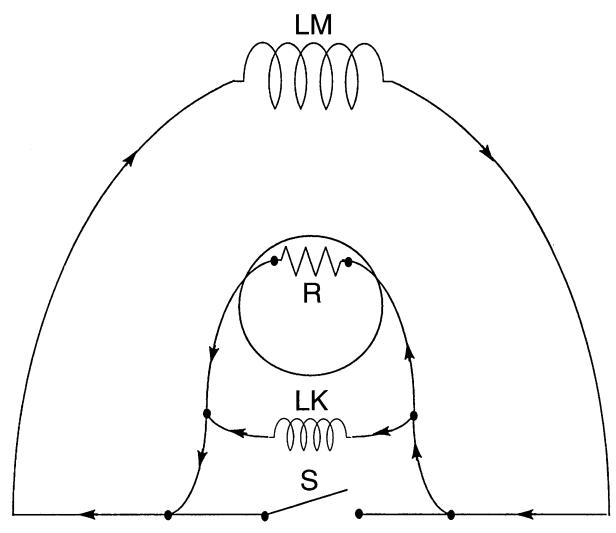

Fig. 1. A perspective view of the substorm expansion phase current system as seen from the north lobe of the tail looking earthward. LM denotes the inductance of the tail current system; $\mathrm{S}$ denotes the switch that opens at the onset of the expansion phase; LK denotes the equivalent inductance of the injected particles (thermal energy formally represented as magnetic energy); (note LK and $\mathrm{S}$ are both in the equatorial plane); and $\mathrm{R}$ denotes the resistance of the ionosphere

The structurally analogous circuit then consists of all four elements in parallel as shown in Fig. 1. The circuit is a modification of the circuit treated in Siscoe (1982). The earlier circuit was designed to represent the growth phase of substorms. Figure 1 gives a possible modification to represent the expansion phase. In any case, it gives a representative circuit analog for the builduprelease model of substorms with which we can follow the flow of energy between circuit components during the expansion phase.

For $t<0$, the switch is closed, and no current flows through $R$ or $L_{K}$. Opening the switch at $t=0$, corresponding to the onset of the expansion phase, shunts the current that formerly circulated only through $L_{M}$ (i.e., some inner portion of the tail current) such that it now flows through $L_{K}$ and $R$ in parallel. This shunted current is the substorm current wedge into which the injected particles $\left(L_{K}\right)$ are placed, since they also receive energy inductively from $L_{M}$. After the transient dies away, a certain amount of energy will have been consumed by the resistor and a residual current will be flowing through $L_{M}$ and $L_{K}$ but not through the resistor. The residual current $\left(I_{f}\right)$ flowing through $L_{K}$ measures the thermal energy (which formally is $1 / 2 L_{K} I_{f}^{2}$ ) that has been added to the ring current as a result of the energy transfer from $L_{M}$. It is readily shown that the ratio of this energy to the energy dissipated in the resistor (i.e., the ratio $\Delta K / \Delta J)$ is $L_{M} /\left(L_{M}+L_{K}\right)$. The expression for the ratio is formally the same ratio found for the capacitors with $L_{M}$ for $C_{1}$ and $L_{K}$ for $C_{2}$. Again we find that $\Delta K / \Delta J$ is always less than unity (for non-zero values of the inductors) independent of the value of the resistor.

The examples show that in the absence of a determination of a statistically significant number of values of $\Delta K / \Delta J$ by means of a self-consistent electrodynamical model, the evidence on which to base expectations available from functionally and structurally analogous lumped circuits suggests that the 
inequality $\Delta K / \Delta J<1$ is a necessary, not contingent, condition.

\section{The role of the growth phase}

The preceding discussion makes plausible the falsity of the original sub-storm (2) hypothesis. If so, we might still be able to rescue the original meaning that the substorm phenomenon is literally a sub-storm by adopting the sub-storm (3) hypothesis. Then we want to measure the change in Dst from before the growth phase to after the expansion phase instead of from just the start of the expansion phase to its end. Now, if we say that the growth phase brings an amount of energy $\Delta M$ into the magnetosphere and the expansion phase then destroys this amount but replaces it with an amount $\Delta K$ of kinetic energy, we see that there is a net gain in storm strength (by the amount $2 \Delta K$ in Eq. 3; the $\Delta M$ term is zero) from before to after a three-phased substorm. In effect the growth phase strengthens the storm more than the expansion phase weakens it, and there is net strengthening.

It is clear, however, that by changing the meanings of words we are wandering here into a semantic no man's land and wish to proceed no farther. There might even be a different way of describing the relation between substorms and storms that avoids the semantic problem altogether. If there is a lesson to be drawn from this observation, it is that in discussing the relation between substorms and storms, one must define one's terms very carefully and not assume that the words mean the same thing to everyone.

\section{Conclusions}

Under two assumptions that seem plausible, substorm expansion phases weaken magnetic storms as measured by -Dst. The assumptions are that substorms expansions are the unloading of magnetic energy stored in the tail and that of the energy thus unloaded more goes into the ionosphere as Joule heat than into the magnetosphere as injected particles. This conclusion lends theoretical plausibility to Iyemori and Rao's (1996) empirical finding that substorm expansions tend to weaken or slow the growth of storms as measured by -Dst.

If substorm expansion phases weaken storms, the sub-storm (2) hypothesis is false, but it might be replaced by a sub-storm (3) hypothesis which preserves the idea that substorms contribute to strengthening the storm.
Acknowledgment. This work was supported in part by the National Science Foundation under grant ATM95-03423.

The Editor-in-chief thanks V.M. Vasyliunas and another referee for their help in evaluating this paper.

\section{References}

Baker, D. N., Driven and unloading aspects of magnetospheric substorms, in Substorms 1, ESA SP-335, 185-191, 1992.

Baker, J., and J. Hurley, A self-consistent study of the Earth's radiation belts, J. Geophys Res., 72, 4351-4355, 1967.

Carovillano, R. L., and G. L. Siscoe, Energy and momentum theorems in magnetospheric dynamics, Rev. Geophys., 11, 289 353, 1973.

Chapman, S., Earth storms: Retrospect and prospect, J. Phys. Soc. Jpn., 17, 6, 1962.

Dessler, A. J., and E. N. Parker, Hydromagnetic theory of geomagnetic storms, J. Geophys. Res., 64, 2239-2252, 1959.

Goertz, C. K., L. -H. Shan, and R. A. Smith, Prediction of geomagnetic activity, J. Geophys. Res., 98, 7673-7684, 1993.

Gonzalez, W. D., J. A. Joselyn, Y. Kamide, H. W. Kroehl, G. Rostoker, B. T. Tsururtani, and V. M. Vasyliunas, What is a geomagnetic storm? J. Geophys. Res., 99, 5771-5792, 1994.

Harel, M., R. A. Wolf, P. H. Reiff, R. W. Spiro, W. J. Burke, F. J. Rich, and M. Smiddy, Quantitative simulation of a magnetospheric substorm: 2. Comparison with observations, J. Geophys. Res., 86, 2242-2260, 1981.

Hesse, M., The magnetotail's role in magnetospheric dynamics: Engine or exhaust pipe? Rev. Geophys., 33, IUGG Supplement, Part 1, 675-683, 1995.

Iyemori, T., and D. R. K. Rao, Decay of the Dst field of geomagnetic disturbance after substorm onset and its implication to storm - substorm relation, Ann. Geophysicae, 14, 608618, 1996.

Kamide, Y., Relationship between substorms and storms, in Dynamics of the Magnetosphere, ed. S.-I. Akasofu, D. Reidel, Dordrecht, pp 425-443, 1980.

Kamide, Y., Is substorm occurrence a necessary condition for a magnetic storm? J. Geomagn. Geoelectr., 44, 109-117, 1992.

Klimas, A. J., D. N. Baker, D. A. Roberts, D. H. Fairfield, and J. Büchner, A nonlinear dynamical analog model of geomagnetic activity, J. Geophys. Res., 97, 12, 253-12, 226, 1992.

McPherron, R. L., Growth phase of magnetospheric substorms, J. Geophys. Res., 75, 5592-5599, 1970.

Olbert, S., G. L. Siscoe, and V. M. Vasyliunas, A simple derivations of the Dessler-Parker-Sckopke relation, J. Geophys. Res., 73, 1115-1116, 1968.

Sckopke, N., A general relation between the energy of trapped particles and the disturbance field over the earth, J. Geophys. Res., 71, 3125-3130, 1966.

Siscoe G. L., The virial theorem applied to magnetospheric dynamics, J. Geophys. Res., 75, 5340-5350, 1970.

Siscoe, G. L., Energy coupling between regions 1 and 2 Birkeland current systems, J. Geophys. Res., 87, 5124-5130, 1982.

Weiss, L. A., P. H. Reiff, J. J. Moss, R. A. Heelis, and B. D. Moore, Energy dissipation in substorms, in Substorms 1. ESA SP-335, 309-317, 1992. 